\title{
Magic-angle theorem in powder x-ray-absorption spectroscopy
}

\author{
R. F. Pettifer \\ Department of Physics, University of Warwick, Coventry CV4 7AL, England \\ C. Brouder \\ Laboratoire de Physique du Solide, Université de Nancy 1, Bôte Postale 239, \\ F-54506, Vandoeuvre-les-Nancy CEDEX, France \\ M. Benfatto and C. R. Natoli \\ Laboratori Nazionali di Frascati dell'Istituto Nazionale di Fisica Nucleare, P.O. Box 13, I-00044 Frascati, Italy \\ C. Hermes \\ EMBL, c/o Deutsches Elektronen Synchroton DESY, Notkestrasse 85, 2000 Hamburg 52, West Germany \\ M. F. Ruiz López \\ Laboratoire de Chimie Théorique, Université de Nancy 1, Bôtte Postale 239, F-54506, Vandoeuvre-les-Nancy CEDEX, France
}

(Received 19 December 1989)

\begin{abstract}
We demonstrate that texture effects can seriously distort the measurement of $x$-ray-absorption data from powdered specimens. We show that the isotropic average dipolar x-ray-absorption spectrum can always be measured irrespective of the degree of texture, provided axial symmetry in the particle distribution function exists normal to the specimen surface and a linearly polarized $\mathrm{x}$-ray beam is employed. A simple modification to synchrotron-radiation experiments to achieve this consists of rotating the specimen by $\theta_{m}$ about an axis perpendicular to the polarization vector and beam direction from normal incidence. $\theta_{m}$ is given by $\sin ^{2} \Theta_{m}=\frac{1}{3}$.
\end{abstract}

\section{INTRODUCTION}

The majority of $\mathrm{x}$-ray-absorption spectra that are currently recorded are of materials that are in powder form, and are measured using synchrotron radiation. The preparation of such specimens is a nontrivial task. ${ }^{1}$ Previously, attention has been focussed on producing homogeneous uniform thickness specimens with $\mu x \approx 1$ ( $x$ is the thickness and $\mu$ the absorption coefficient), which minimizes distortion to the spectra resulting from the finite bandwidth of the monochromator and inhomogeneous thickness effects. To this list of requirements should be added a knowledge of texture effects (preferred orientation). It has been known for many years that $\mathrm{x}$ ray absorption spectra exhibit orientational effects for non-cubic materials, even for an unpolarized $\mathrm{x}$-ray beam, ${ }^{2,3}$ since the electric field vector $\widehat{\varepsilon}$ must be transverse to the direction of propagation. Further, it is also well known that powder $\mathrm{X}$-ray diffraction is severely distorted from the ideal isotropic case by texture effects which are difficult to avoid. ${ }^{4}$ No general technique is known to eliminate textural effects in x-ray diffraction although it is known that reducing the particle size greatly reduces the extent of texture. Unfortunately this is not always possible especially when dealing with delicate crystals. X-ray diffraction samples a similar depth of specimen as that observed in X-ray absorption. Thus it is logical to conclude that texture effects may be a problem in $\mathrm{x}$-ray absorption also. It is frequently assumed with powders that they exhibit no texture and are analyzed accordingly. Thus the presence of texture may well lead to confusion, either when comparing a standard with an unknown material, or when performing $a b$ initio calculations.

In Sec. III of this paper, we demonstrate the effects of texture experimentally using specimens of the superconductor $\mathrm{La}_{1.85} \mathrm{Ba}_{0.15} \mathrm{CuO}_{4}$ prepared by conventional techniques. In Sec. II we show from symmetry arguments that, under conditions which normally prevail for specimen preparation, texture effects can be totally avoided for dipole transitions by employing the results of a magic-angle theorem.

\section{THEORY}

We prove the magic-angle theorem for powder $\mathrm{x}$-ray absorption spectroscopy in two ways. First, in Sec. II A we establish that the absorption cross section transforms as a second rank Cartesian tensor from microscopic theory provided the transitions are dipolar. We then exploit the symmetry properties of the particle distribution function to find the average tensor for the specimen. In Sec. II B we provide a more rigorous proof based on the analysis of polarization absorption effects given recently. ${ }^{5}$

\section{A. Magic-angle theorem using Cartesian tensors}

Starting from the Golden Rule for absorption, and considering only dipole transitions we can quickly estab- 
lish that the polarization dependent absorption cross section can be written in atomic units as

$$
\begin{aligned}
\sigma(\widehat{\boldsymbol{\varepsilon}}) & =4 \pi^{2} \alpha \omega \sum_{f}|(f|\boldsymbol{\varepsilon} \cdot \mathbf{r}| i)|^{2} \delta\left(\omega+E_{i}-E_{f}\right) \\
& =\sum_{i, j=1}^{3} \varepsilon_{i} \varepsilon_{j} \sigma_{i j}(\omega)
\end{aligned}
$$

where $\varepsilon_{i}$ is a Cartesian component of the linear polarization vector and the elements of the array $\sigma_{i j}$ are given by

$$
\begin{aligned}
\sigma_{i j}(\omega)=4 \pi^{2} \alpha \omega \frac{1}{2} \sum_{f} & {\left[\left(i\left|x_{i}\right| f\right)\left(f\left|x_{j}\right| i\right)\right.} \\
& \left.+\left(i\left|x_{j}\right| f\right)\left(f\left|x_{i}\right| i\right)\right] \delta\left(\omega+E_{i}-E_{f}\right) .
\end{aligned}
$$

This definition of $\sigma_{i j}$ shows the antisymmetric imaginary array elements, which are not observed with linearly polarized radiation. As usual, in Eq. (1) $\alpha=\frac{1}{137}$ is the fine structure constant, $\omega$ is the photon energy, $E_{i}, E_{f}$ are the energies of the initial and final states, respectively, and for brevity

$$
\mathbf{r}=\sum_{k=1}^{N} \mathbf{r}_{k},
$$

where the sum is over all the $N$ electrons in the system. Clearly from Eq. (2) $\sigma_{i j}$ form the elements of a symmetric array, ${ }^{6}$ and transform according to the product of two vectors having defined a Cortesion tensor of rank 2 .

The above applies to any crystal, but now we focus attention on a subset of crystallites which have a plane $(h k l)$ parallel to the composite sample surface. Selecting one crystallite from this subset we define two sets of axes. One set is bound to the sample frame with the $z$ axis normal to the sample surface. The other set of axes is coincident with the principal axes of the crystallite. We can thus transform the tensor referred to its principal axes to its components in the sample frame via the tensor transformation law:

$$
\sigma_{i j}=\sum_{k l} R_{k i} R_{l j} \sigma_{k l}
$$

where the rotation matrices are given in terms of the Euler angles $\alpha, \beta, \gamma$ using the convention of Ref. 7 as

$$
R=\left(\begin{array}{ccc}
\cos \alpha \cos \beta \cos \gamma-\sin \alpha \sin \gamma & -\cos \alpha \cos \beta \sin \gamma-\sin \alpha \cos \gamma & \cos \alpha \sin \beta \\
\sin \alpha \cos \beta \cos \gamma+\cos \alpha \sin \gamma & -\sin \alpha \cos \beta \sin \gamma+\cos \alpha \cos \gamma & \sin \alpha \sin \beta \\
-\sin \beta \cos \gamma & \sin \beta \sin \gamma & \cos \beta
\end{array}\right)
$$

To find the tensor which represent the average cross section of our subset of crystallites corresponds to averaging over the angle $\gamma$, as each crystallite is free to rotate about the sample normal while retaining the given $(h k l)$ plane parallel to the sample surface. The result is the tensor with components in the sample frame:

$$
\langle\sigma(\alpha, \beta)\rangle_{\gamma}=\left(\begin{array}{ccc}
\sigma_{\perp}(\alpha, \beta) & 0 & 0 \\
0 & \sigma_{\perp}(\alpha, \beta) & 0 \\
0 & 0 & \sigma_{\|}(\alpha, \beta)
\end{array}\right)
$$

where

$$
\begin{aligned}
\sigma_{1}(\alpha, \beta)= & \frac{1}{2}\left(\cos ^{2} \alpha \cos ^{2} \beta+\sin ^{2} \alpha\right) \sigma_{11} \\
& \left.+\frac{1}{2} \sin ^{2} \alpha \cos ^{2} \beta+\cos ^{2} \alpha\right) \sigma_{22}+\frac{1}{2} \sin ^{2} \alpha \sigma_{33}
\end{aligned}
$$

and

$\sigma_{\|}(\alpha, \beta)=\left(\cos ^{2} \alpha \sin ^{2} \beta\right) \sigma_{11}+\left(\sin ^{2} \alpha \cos ^{2} \beta\right) \sigma_{22}+\cos ^{2} \beta \sigma_{33}$.

We note that the tensor representing the average property of the subset of crystallites has its principal axes in the sample plane. This result is true regardless of the choice of plane $(h k l)$, and so is true in general. The details of the texture can now be included in the argument as weighting coefficients to the tensor above in the grand average of all crystallites in the specimen. The result however is still a tensor whose principal axes lie along the sample normal which we express as

$$
\sigma(\widehat{\boldsymbol{\varepsilon}})=\sigma_{a} \sin ^{2} \theta+\sigma_{b} \cos ^{2} \theta,
$$

where $\theta$ is the angle between the polarization vector and the sample normal. $\sigma_{a}$ and $\sigma_{b}$ contain the details of the distribution function of crystallite orientations. If $\theta$ is now chosen such that $\cos ^{2} \theta_{c}=\frac{1}{3}$ then

$$
\sigma(\theta)=\left(2 \sigma_{a}+\sigma_{b}\right) / 3=\bar{\sigma},
$$

and we obtain the isotropic average. Thus, we note that the details of the particle distribution function do not enter our argument; the isotropic cross section will always be measured provided only that the particle distribution function has a $C_{\infty}$ axis perpendicular to the sample surface. Also it should be noted that the specific transitions involved do not enter the argument and thus the result holds for any absorption edge. Curved-wave, multiple-scattering and many-body effects are also implicitly included. We have assumed, however, that the cross section of the composite sample is the superposition of the cross sections of the individual crystallites. This should be true provided that the intragrain interactions dominate the intergrain influences. This will be a good approximation provided grains of at least $100 \AA$ are assumed. We should also note that it is necessary to restrict our attention to dipole transitions as these transform as the product of two vectors and thus can be represented as a tensor of rank 2. Electric quadrupole transitions transform as the product of four vectors and thus the result is no longer valid.

We should note here that absorption specimens which possess rotational symmetry about the sample normal are quite easy to obtain. If we consider, for example, the 
casting of specimens, then the single rotation axis is provided by gravity. This controls the selection of cleavage planes which lie preferentially perpendicular to this axis. A possible exception may occur if interparticle interactions are not small (e.g., electrostatic or magnetic). When pressing powders, the rotation axis will be along the direction of uniaxial stress as the flow lines will also have rotational symmetry.

\section{B. Direct proof of the magic-angle theorem using the spherical tensor property of the cross section}

It has been shown recently ${ }^{5}$ that the general dipole polarization-dependent absorption cross section for linearly polarized radiation can be written as

$$
\sigma(\widehat{\boldsymbol{\varepsilon}})=\sigma(0,0)-\left[\frac{8 \pi}{5}\right]^{1 / 2} \sum_{m=-2}^{2} Y_{2}^{m}(\widehat{\boldsymbol{\varepsilon}}) \sigma(2, m)
$$

where $\sigma(0,0)$ and $\sigma(2, m)$ are spherical tensors of rank 0 and 2 , respectively. The first term is the isotropic cross section, whereas the second term gives the angular dependance. If $\underline{R}$ is the rotation matrix which transforms the sample reference frame into the crystal reference frame then the sample normal $\hat{\mathbf{n}}$ in the crystal reference frame is given by

$$
\widehat{\mathbf{n}}=\left(\begin{array}{c}
\sin \theta \cos \phi \\
\sin \theta \sin \phi \\
\cos \theta
\end{array}\right)=\underline{R}^{-1}\left(\begin{array}{l}
0 \\
0 \\
1
\end{array}\right) .
$$

Using the Euler angle convention of Ref. 7 we can identify $\alpha=\phi$ and $\beta=\theta$. Transforming $\sigma(2, m)$ into the sample frame gives

$$
\widetilde{\sigma}(2, m)=\sum_{m^{\prime}=-2}^{2} D_{m^{\prime} m}^{2}\left(\underline{R}^{-1}\right) \sigma\left(2, m^{\prime}\right),
$$

where we note that the tensor components transform with $\underline{R}^{-1}$ if the vector components transform with $\underline{R}$ (Ref. 7). The Wigner matrix can be written

$$
D_{m^{\prime} m}^{2}(\alpha, \beta, \gamma)=e^{-i m^{\prime} \alpha} \alpha_{m^{\prime} m}^{2}(\beta) e^{-i m \gamma}
$$

averaging over $\gamma$ as the crystallite is free to rotate about the $z$ axis in the sample frame gives

$$
\begin{aligned}
\left\langle D_{m^{\prime} m}^{2}(\alpha, \beta, \gamma)\right\rangle_{\gamma} & =D_{m^{\prime} m}^{2}(\alpha, \beta, 0) \delta_{m 0} \\
& =D_{m^{\prime} 0}^{2}(\phi, \theta, 0) \delta_{m 0}
\end{aligned}
$$

so the average dipole absorption cross-section tensor components are

$$
\langle\widetilde{\sigma}(2, m)\rangle_{\gamma}=\sum_{m^{\prime}=-2}^{2} D_{m^{\prime} 0}^{2}(\phi, \theta, 0) \sigma\left(2, m^{\prime}\right) \delta_{m 0},
$$

and thus

$$
\begin{aligned}
\langle\sigma(\widehat{\boldsymbol{\varepsilon}})\rangle_{\gamma} & =\sigma(0,0)-\left[\frac{8 \pi}{5}\right]^{1 / 2} Y_{2}^{0}(\widehat{\boldsymbol{\varepsilon}})\langle\widetilde{\sigma}(2,0)\rangle \\
& =\sigma(0,0)-\sqrt{2} P_{2}\left(\theta_{\varepsilon}\right)\langle\widetilde{\sigma}(2,0)\rangle .
\end{aligned}
$$

In other words, for a given crystallographic plane, the average of the photoabsorption cross section over the

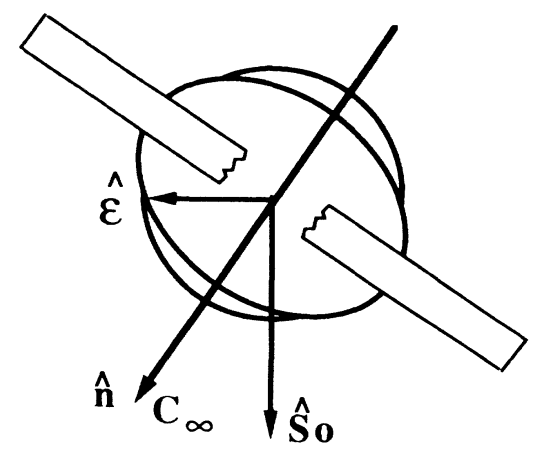

FIG. 1. Schematic diagram of an x-ray absorption specimen. $\widehat{s_{0}}$ is the incident beam vector, $\hat{\mathbf{n}}$ is the normal to the specimen which coincides with a $C_{\infty}$ rotation axis. $\hat{\varepsilon}$ is the polarization vector which points to a circle on the representation quadric ellipsoid which intersects the isotropic representation quadric sphere. The angle between $\widehat{\mathbf{s}}$ and $\hat{\mathbf{n}}$ is $\theta_{m}$ and the angle between $\hat{\mathbf{n}}$ and $\hat{\boldsymbol{\varepsilon}}$ is $\theta_{c}$.

crystallite orientations in that plane is equal to the isotropic absorption when the angle between the polarization vector and the sample normal is equal to the angle given by $\cos ^{2} \theta=\frac{1}{3}$. Of course, in a real sample, the crystallites sit on various planes, but this does not change the result, since this is true for an arbitrary plane.

\section{General statement of the magic-angle theorem}

The foregoing proofs can be summarized by stating the result as a theorem which we express in terms of $\theta_{m}$ which is the compliment of $\theta_{c}$ the angle used immediately above. This is because it is more natural for the experimentalist to measure the angle of incidence of the x-ray beam onto the sample rather than its compliment. The magic-angle theorem can be stated thus:

For a powdered homogeneous x-ray absorption specimen which possesses axial symmetry in its particle distribution function about an axis which lies along the sample normal, then it is always possible to measure the isotropic average $\mathrm{x}$-ray dipole absorption spectrum provided linear polarization is used and the sample normal is rotated by an angle $\theta_{m}$ from the beam direction about an axis perpendicular to the beam and the polarization vector. $\theta_{m}$ is given by $\sin ^{2} \theta_{m}=\frac{1}{3}$.

The situation is sumarized in Fig. 1.

\section{EXPERIMENTAL}

\section{A. Powder specimens}

We used the material $\mathrm{La}_{1.85} \mathrm{Ba}_{0.15} \mathrm{CuO}_{4}$ in order to test the above theorem experimentally as we can anticipate from its crystal structure that the $\mathrm{x}$-ray absorption spectrum (EXAFS and XANES) will be highly anisotropic. Indeed, XANES calculations ${ }^{8}$ have demonstrated this.

Two specimens were prepared from the same batch of material. Specimen A was prepared by grinding the material to a powder in a pestle and mortar. Specimen $\mathbf{B}$ was similarly treated but was then subjected to a further milling operation in a vibrating roller mill. Both powders 


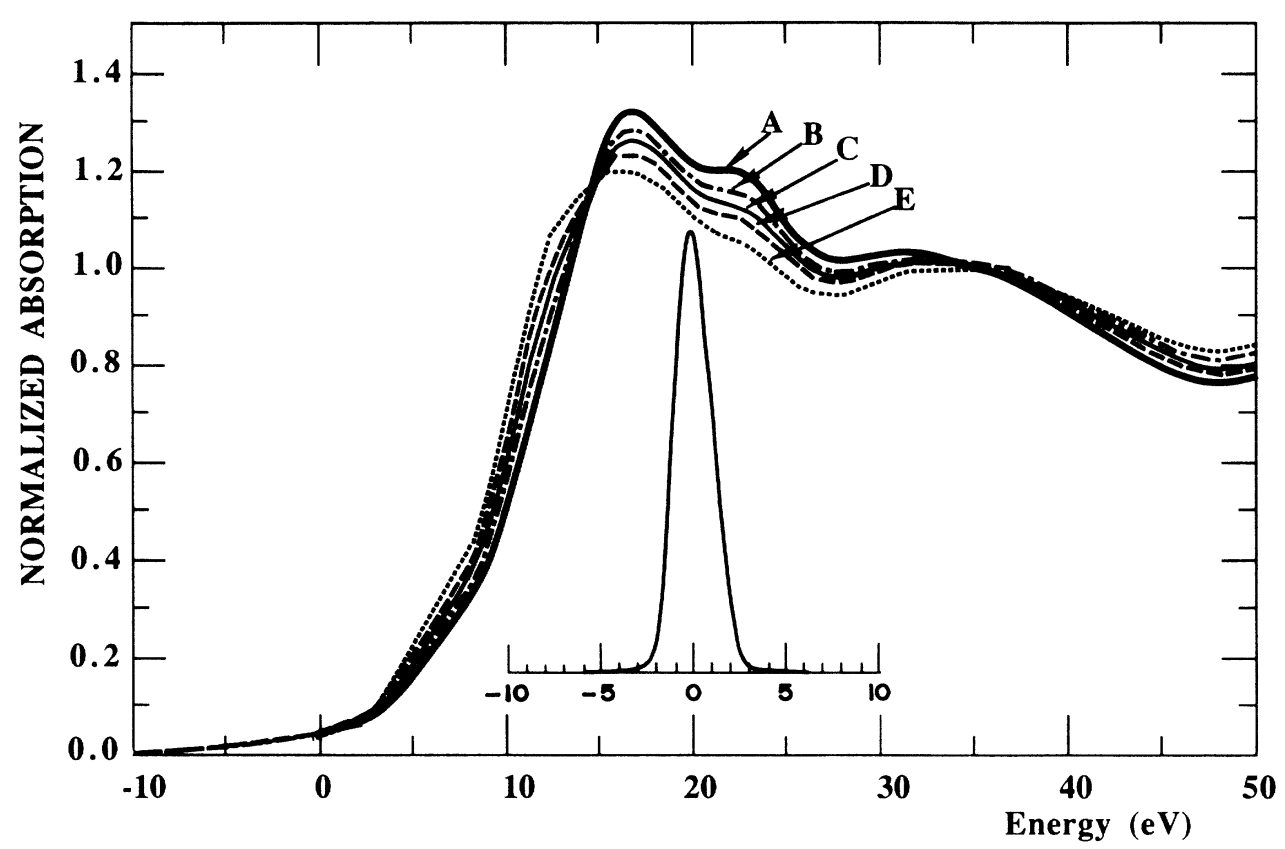

FIG. 2. The normalized $K$ edge of copper in $\mathrm{La}_{1.85} \mathrm{Ba}_{0}{ }_{15} \mathrm{CuO}_{4}$ for specimen $\mathrm{A}$ as a function of angle of rotation about an axis normal to the plane containing the polarization and beam directions. The angle is measured between the sample normal and the beam direction. The energy axis is referred to $8980.6 \mathrm{eV}$ which is the first inflection point of the copper metal edge. Measurements were recorded at the following angles: $A\left(0^{\circ}\right), B\left(26^{\circ}\right), C\left(32^{\circ}\right), D\left(42^{\circ}\right)$, and $E\left(56^{\circ}\right)\left( \pm 3^{\circ}\right)$. The inset shows the instrument function for these measurements.

were mixed separately with celulose nitrate glue which had been diluted with amylacetate. The individual specimens were then cast onto a flat glass plate ensuring that the gravitational axis was perpendicular to the plate. Following the $x$-ray absorption measurements the powders in the samples were examined in a scanning elec- tron microscope. Specimen A was found to contain grains whose aspect ratio was typically $4: 1$ and these platelets had their largest dimension typically $20 \mu \mathrm{m}$ but spanning a range $40 \mu \mathrm{m}$ to approximately $5 \mu \mathrm{m}$. Specimen B however had its largest side with typical dimensions $5 \mu \mathrm{m}$ but still exhibiting aspect ratios of about $4: 1$.

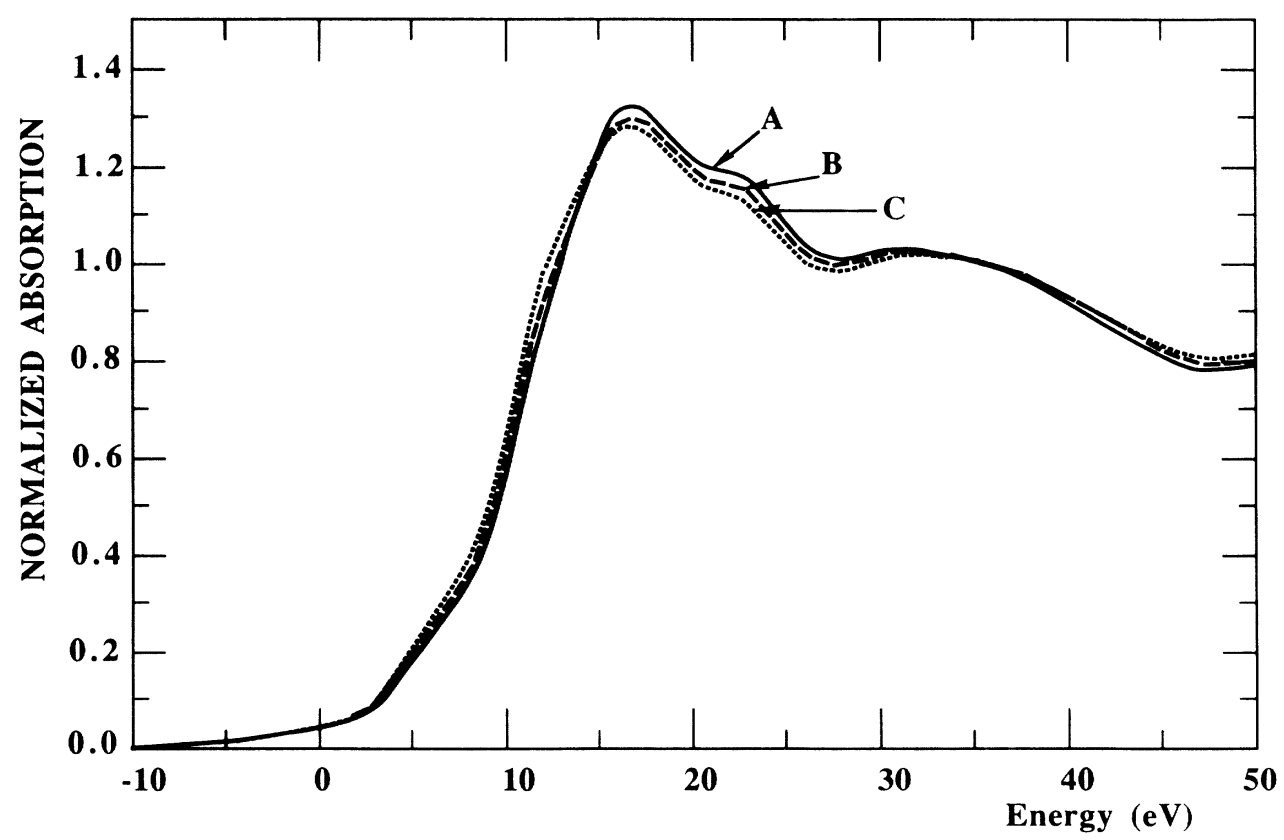

FIG. 3. The normalized $K$ edges as in Fig. 2 but for the fine particle specimen B. Measurements were taken at the following angles: $A\left(0^{\circ}\right), B\left(32^{\circ}\right)$, and $C\left(45^{\circ}\right)$. 


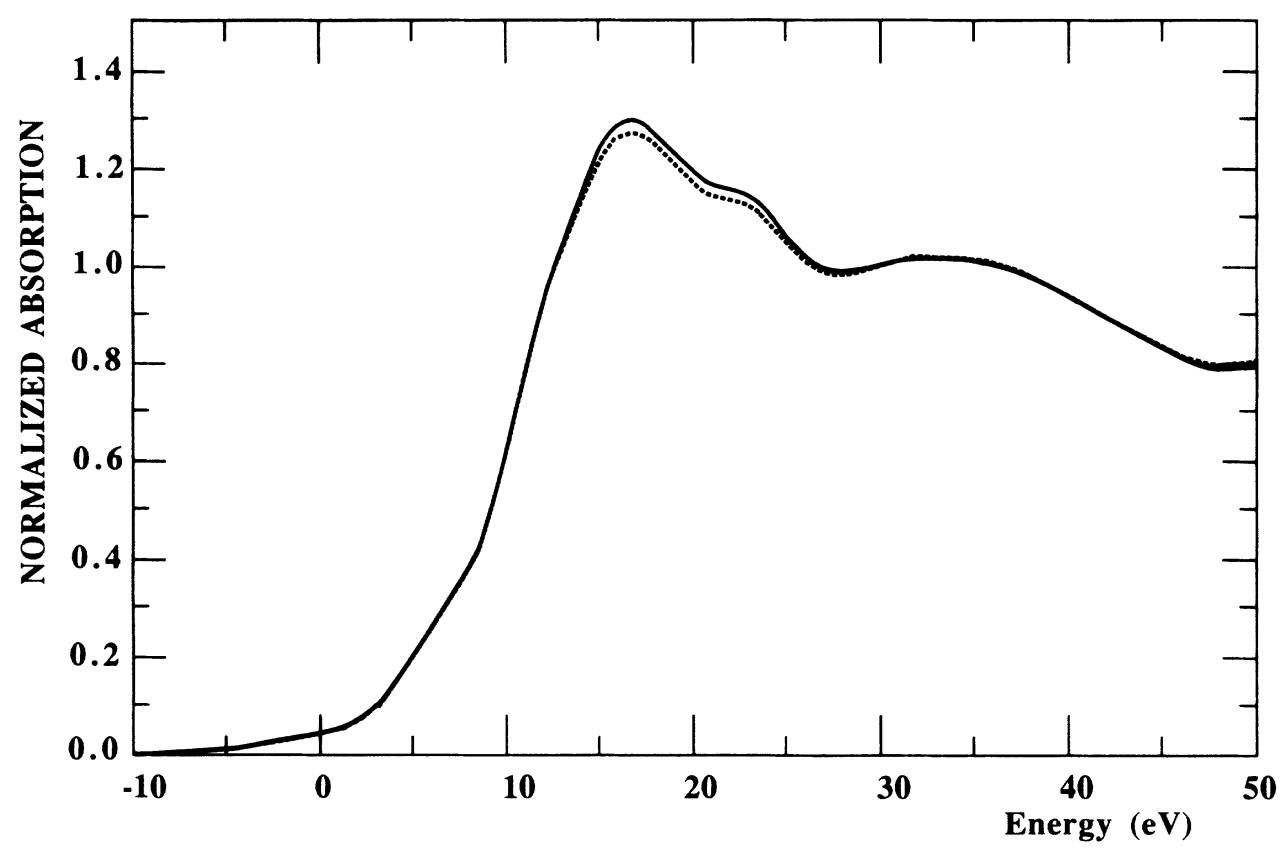

FIG. 4. The normalized $K$-edge spectra of samples B (solid) and A (dashed) recorded at the magic angle of $35.26^{\circ}\left( \pm 3^{\circ}\right)$.

\section{B. X-ray absorption measurements}

The $\mathrm{x}$-ray absorption measurements were all performed on the EMBL EXAFS beamline in HASYLAB using radiation from the storage ring DORIS II operating at 3.7 $\mathrm{GeV}$. The crystal monochromator was $\mathrm{Si}(111)$ mounted as described previously. ${ }^{9}$ The spectra were absolutely calibrated in $\mathrm{x}$-ray energy by using a calibrator ${ }^{10}$ which allowed the instrument function to be determined; this is shown as an inset in Fig. 2. Prior to the measurments being taken, the entire instrument was aligned to accept 3 $\mu$ rads spanning the central portion of the vertical radiation fan from DORIS II. This alignment was maintained via a feedback system to the electron orbit. ${ }^{11}$ Integrating the vertical beam using the full beam optic parameters for our line $(D)$, yielded a polarization state of $>98 \%$ for the horizontal polarization component.

The spectra were recorded by scanning coarsely from $400 \mathrm{eV}$ below the edge to $1400 \mathrm{eV}$ above the edge, with the region shown in Fig. 2 measured finely. All measurements were made at $302 \mathrm{~K}$. This enabled the spectra to be normalized by fitting a Victoreen $\left(\mu=A E^{-3}+B E^{-4}\right)$ relationship in the pre-edge region and a second degree polynomial to the post-edge region. The absorption edge jump at the copper $K$ edge was $\Delta \mu x=0.4$ and 0.15 for normal incidence on specimens $A$ and $B$, respectively. Figure 2 shows the variation of the normalized sample absorption as a function of angle of incidence and clearly demonstrates the presence of texture for specimen $\mathrm{A}$. Figure 3 gives a similar plot for sample $B$. The feature at $17 \mathrm{eV}$ exhibits a variation of 2.3 and $1.2\left(\times 10^{-3} \mathrm{deg}^{-1}\right)$ for samples A and B, respectively, clearly demonstrating a different texture in both samples.

Both specimens were then oriented at the magic angle and the spectra recorded. The results are shown in Fig.
4. The shape of both curves is well reproduced although the amplitude of the structure for the coarse specimen (A) is reduced with respect to that of the fine material (B). The most likely cause for this is the increased particle inhomogeneity in specimen A. Despite this, the subtle shoulder at $12 \mathrm{eV}$ is reproduced in both specimens. This shoulder is not in evidence for the normal incidence measurements of both specimens. It should also be noted that for these specimens the effects of texture cause a larger distortion to the measured spectra than specimen inhomogeneities. The difference in fine-structure amplitude between spectra measured at the magic-angle and normal incidence is $7.8 \%$ and $4.3 \%$ for specimens $\mathrm{A}$ and B.

\section{DISCUSSION}

We have demonstrated that large textural effects are observable in $\mathrm{x}$-ray absorption spectra owing to the polarization dependence of the absorption cross section. These effects can however be removed by using a magicangle technique described in this paper, provided that the particle distribution function has rotational symmetry about the sample normal and a linearly polarized $\mathrm{x}$-ray beam is available. Consequently, this is a special property of synchrotron radiation where the polarization state can be well defined and linear.

There is some similarity between our result and the magic-angle theorem applied to nuclear magnetic resonance experiments, especially in terms of the mathematics employed, and the inevitable appearance of the second Legendre polynomial. However, our result refers to a static average and we deal only with the single polarization vector, while the former averages dynamically over two vectors. 
While in this paper we concentrate on using the symmetry properties of the particle distribution and neglect any symmetry properties possessed by the individual crystallites themselves in order to make the results completely general. However, we should remark that crystals belonging to the cubic class while possessing cleavage planes and texture will not of course exhibit anisotropic $\mathrm{x}$-ray absorption structure (except for the small quadrupole contribution). Thus we are specifically concerned with noncubic materials.

It has been argued ${ }^{3,12}$ previously, on the basis of classical electromagnetic theory, that the x-ray absorption cross-section transforms as a second rank Cartesian tensor. However, classical theory cannot be used in the $\mathrm{x}$ ray regime. ${ }^{13}$ The result is only true for dipole transitions. This does not however negate the conclusions drawn by others, but our result justifies them with the restriction to dipole transitions only.

The specimen preparation technique used for specimens A and B is frequently employed by experimentalists and it is possible that texture effects may be partially responsible for the apparent absence of scattering amplitude transferability between differing materials. In most cases, the almost trivial modification to the experimental technique can easily be employed to remove this source of error. The change in the absorption experienced in employing the magic-angle technique $\mu x\left(\theta_{m}\right) / \mu x(0)$ $=1.225$ should cause no problems.

\section{CONCLUSIONS}

Textures effects may be important in x-ray absorption measurements. However these effects may be avoided by employing a magic-angle theorem. This results in a relatively simple modification to experimental technique for measurements using a synchrotron source.

\section{ACKNOWLEDGMENTS}

We would like to thank G. Balakrishnan and D. Paul for providing samples of the material used in this work. Also one of us (R.F.P.) would like to thank European Molecular Biology Laboratory (EMBL) and European Molecular Biology Organization (EMBO) for financial support, and the University of Nancy 1 and Laboratori Nazionali di Frascati dell'Istituto Nazionale di Fisica Nucleare (INFN) for their hospitality. We would like to thank the following for helpful discussions or critical reading of the manuscript: D. Pflugge, H. Mykura, G. Smith, and G. Krill. The Science and Engineering Research Council (SERC) are acknowledged for the provision of computing facilities.
${ }^{1}$ H. Nolting, W. Tremel, P. Eggars, G. Henkel, and B. Krebs, Nucl. Instrum. Methods A259, 576 (1987), and references therein.

${ }^{2}$ P. A. Lee and J. B. Pendry, Phys. Rev. B 11, 2795 (1975).

${ }^{3}$ S. M. Heald and E. A. Stern, Phys. Rev. B 16, 5549 (1977), and references therein.

${ }^{4}$ H. P. Klug and L. E. Alexander, X-ray Diffraction Procedures, 2nd ed. (Wiley, New York, 1975), p. 365.

${ }^{5}$ The latter results from simplifications to the theory in $\mathrm{C}$. Brouder, M. F. Ruiz Lopez, R. F. Pettifer, M. Benfatto, and C. R. Natoli, Phys. Rev. B 39, 1488 (1989).

${ }^{6} \mathrm{~J}$. Nye, The Physical Properties of Crystals and their Representation by Tensors and Matrices (Oxford University Press, Ox- ford, England, 1957), p. 13.

${ }^{7}$ L. C. Biedenham and J. D. Louck, in Encyclopedia of Mathematics and its Applications (Addison-Wesley, Reading, MA, 1981).

${ }^{8}$ P. J. Durham (unpublished).

${ }^{9}$ R. F. Pettifer and C. Hermes, J. Phys. (Paris) C 8, 127 (1986).

${ }^{10}$ R. F. Pettifer and C. Hermes, J. Appl. Crystallogr. 18, 404 (1985).

${ }^{11}$ W. Brefeld, Nucl. Instrum. Methods A261, 22 (1987).

${ }^{12}$ E. A. Stern, in $X$-ray Absorption, edited by D. C. Koningsberger and R. Prins (Wiley, New York, 1988), p. 26.

${ }^{13}$ J. D. Jackson, Classical Electrodynamics (Wiley, New York, 1975), p. 227. 